Aloma 2020, 38(2), 121-136

Revista de Psicologia, Ciències de l'Eduació i de l’Esport

ISSN: $1138-3194$

CFacultat de Psicologia, Ciències de l'Educació i de l’Esport Blanquerna

Universitat Ramon Llull

cc)(1) (2)

BY NC SA

\title{
Test adaptativos informatizados, pruebas computarizadas y pruebas en aplicaciones móviles: comparación de su disponibilidad actual en Latinoamérica, Estados Unidos y Canadá
}

\author{
Joel Figueroa-Quiñones ${ }^{1}$, Ray Moncada Oliva ${ }^{1}$, Carlos Alberto Coronado Zapata ${ }^{1}$ \\ \& Juan Carlos Bazo-Alvarez ${ }^{1,2}$ \\ 'Universidad Católica Los Ángeles de Chimbote, Instituto de Investigación, Chimbote, Perú \\ 2 PSYCOPERU - Instituto de Investigación, Capacitación y Desarrollo Psicosocial y Educativo, Lima, Perú
}

Recibido: 2020-06-24

Aceptado: 2020-10-09

Test adaptativos informatizados, pruebas computarizadas y pruebas en aplicaciones móviles: comparación de su disponibilidad actual en Latinoamérica, Estados Unidos y Canadá

\begin{abstract}
Resumen. Las pruebas psicométricas han cambiado su formato de lápiz y papel a evaluaciones informatizadas progresivamente en los últimos 40 años, debido a que el formato informatizado ofrece varias ventajas sobre el clásico. En este estudio comparamos la producción y disponibilidad de los test adaptativos informatizados (TAI), las pruebas computarizadas (PC) y las pruebas en aplicativos móviles (PAM) entre Estados Unidos, Canadá y Latinoamérica. Realizamos una revisión narrativa, explorando bases de datos como PsycINFO, Scopus, Web of Science, PubMed y SciELO. Para complementar los hallazgos, realizamos una búsqueda en Google y Play Store. Los resultados mostraron que Estados Unidos y Canadá registran la mayor producción y disponibilidad de TAI, PC y PAM. En ambas regiones, la mayor parte de los TAI y las PC está diseñada para evaluar a los adultos, mientras que las PAM están diseñadas para cualquier edad. En Estados Unidos y Canadá, los TAI y las PC son en gran parte originales y su uso requiere de un pago previo, mientras que en Latinoamérica los TAI, las PC y las PAM suelen ser versiones adaptadas y de acceso libre. Finalmente, existe una brecha amplia en la disponibilidad de TAI, PC y PAM en ambas regiones. Estados Unidos y Canadá tienen a su disposición más de trece veces la cantidad de TAI, más del doble de cantidad de PC y más del quíntuple de cantidad de PAM que en Latinoamérica. Concluimos que existe una gran brecha en cuanto a la producción y el acceso a instrumentos psicométricos de alta tecnología que Latinoamérica necesita superar. Finalmente, proveemos un listado de todos los TAI, las PC y las PAM disponibles actualmente, de acceso gratuito y de pago.
\end{abstract}

Palabras clave: Pruebas computarizadas; pruebas en aplicativos móviles; test adaptativos informatizados

Computerized adaptive tests, computerized tests and mobile applications: comparison of their current availability in Latin America and the United States/Canada

\begin{abstract}
Summary. Psychometric tests have changed their pencil and paper format into computerized evaluations progressively in the last 40 years, since the computerized format offers several advantages over the classic one. In this study, we compared the production and availability of computerized adaptive tests (CAT), computerized tests (CT) and tests in mobile applications (TMA), between the United States/Canada and Latin America. We conducted a narrative review, exploring databases such as PsycInfo, Scopus, Web of Science, Pubmed, and Scielo. To complement the findings, we conducted a search on Google and the Play Store. The results showed that the United States/Canada records the highest production and availability of CAT/CT/TMA. In both regions, most of CAT/CT are designed to evaluate adults, while TMA are designed for any age. In the United States/Canada, CAT/CT are mostly original, and their use requires a previous payment, while in Latin America, CAT/CT/TMA are usually adapted and freely available versions. Finally, there is a huge difference in the availability of CAT/CT/TMA between both regions. The United States/ Canada has at its disposal more than thirteen times the amount of CAT, more than double the amount of CT and more than five times the amount of TMA than Latin America. We concluded that there is a large gap in production and access to high-tech psychometric instruments that Latin America needs to overcome. Finally, we provide a list of all CAT/CT/TMA currently available, free or paid access.
\end{abstract}

Keywords: Computerized adaptive test (CAT); computerized tests; tests in mobile applications

Correspondencia

Juan Carlos Bazo-Alvarez

Universidad Católica Los Ángeles de Chimbote,

Instituto de Investigación, Chimbote, Perú

juan.bazo.a@gmail.com 


\section{Introducción}

Las pruebas psicométricas han sido utilizadas desde su aparición a inicios del siglo xx mayormente en el típico formato de lápiz y papel (Coulacoglou \& Saklofske, 2018). Sin embargo, con el avance de las tecnologías de la información (v.g. la masificación de las computadoras e Internet), desde finales de los setenta del siglo pasado, el típico formato fue paulatinamente cambiando por el nuevo formato digital. Estas versiones digitales fueron conocidas como pruebas computarizadas (PC), la primeras dirigidas a evaluar aspectos cognitivos como la memoria (Olea, Abad \& Barrada, 2010). Las PC se caracterizan por automatizar la aplicación, acelerar el proceso de calificación y enriquecer la interpretación con indicadores propios del recurso informático (Sternin, Burns \& Owen, 2019). Por ejemplo, las pruebas basadas en tareas pueden tener un registro muy preciso del tiempo de ejecución gracias a la computadora. Particularmente, la evaluación simultánea de grandes grupos, lo mismo que las evaluaciones en forma remota, se volvieron mucho más viables gracias a las PC y a Internet.

Los test adaptativos informatizados (TAI) son instrumentos electrónicos de evaluación que, a diferencia de las PC, se adaptan a las características del evaluado durante la aplicación de la prueba (Burga León, 2019). Los TAI funcionan con un algoritmo que selecciona preguntas a partir de un banco de ítems o preguntas diferentes, la selección se adapta de manera progresiva de acuerdo al nivel de competencia, rendimiento o característica reflejado en cada respuesta del evaluado (Graham et al., 2019; Olea et al., 2010). Adicionalmente a su facilidad de calificación automática, atributo que comparte con las PC, los TAI brindan una mayor precisión de medida (mayor confiabilidad) (Olea, Ponsoda \& Prieto, 1999). Esto se logra gracias al algoritmo que hay detrás del TAI. Por ejemplo, en un test de conocimientos, un TAI selecciona cada pregunta a partir de un banco de ítems calibrados según su dificultad, y el algoritmo elige el siguiente ítem como una pregunta más fácil si el evaluado falla, o una más difícil si acierta. De esta manera, el test se "adapta" a las características propias del evaluado, lo que permite maximizar la confiabilidad de la medida del atributo o rasgo. Esta adaptación implica que, rara vez, dos evaluados respondan a las mismas preguntas, lo que hace menos viable que se entrenen para un TAI específico o se copien durante su ejecución. Otras características, como la elaboración de informes automatizados y la reducción del tiempo de calificación, son compartidas también con las PC (Rojas, 2001; Van Horn, 2003).

La primera generación de TAI consistió en diseños dirigidos a evaluar solo aspectos del rendimiento educativo y el vocabulario (Rojas, 2001). En países como Estados Unidos, Taiwán y Holanda, comenzaron a perfeccionar el desarrollo de TAI para un uso más generalizado en la medición y evaluación de diversas áreas de la salud, psicología y educación; tales como la calidad de vida (Turner-Bowker et al., 2012), indicado- res de salud mediante el monitoreo ambulatorio (Rose et al., 2012; Wang, Dai \& Ding, 2010), el comportamiento adictivo (Ferreri et al., 2018), el organizacional (Barney \& Fisher, 2016) y la evaluación de la lectura y las competencias educativas (Lunz, Bergstrom \& Wright, 1992; Shamir, 2018).

Las pruebas en aplicativos móviles (PAM) son otro formato digital que ha ganado popularidad entre los usuarios durante los últimos años. Las PAM comparten algunas características similares a las PC y a los TAI, como la calificación automática e interpretación de resultados inmediatos. Sin embargo, las PAM difieren por su facilidad de acceso al usuario, pues éstas pueden descargarse y operarse fácilmente desde una tableta o un teléfono móvil (Bakker et al., 2016).

Las pruebas en todas estas versiones digitales han sido diseñadas para la evaluación de distintas poblaciones, como niños (Huang et al., 2018), jóvenes universitarios (Kalender \& Berberoglu, 2017), adultos mayores (Gifford, Liu, Romano, Jones \& Jefferson, 2015) y personas enfermas o con discapacidad física (Lee et al., 2018). A pesar de su importancia para la evaluación psicológica, no existe a la fecha una exploración minuciosa de la disponibilidad de las PAM, las PC y los TAI en el contexto Latinoamericano.

El desarrollo de PAM, PC y especialmente de TAI puede generar costos adicionales relativos a la tecnología que emplean. Por ejemplo, la adquisición de software especializado implica costos que pueden ser elevados, lo mismo que las constantes actualizaciones de software que hacen posible la administración de estas pruebas en nuevos sistemas operativos (Sierra et al., 2009). La calibración de amplios bancos de ítems requiere de largos periodos de análisis y recursos humanos especializados para su ejecución. Asimismo, durante el diseño y desarrollo de un TAI, pueden surgir dificultades técnicas y de logística en los laboratorios de cómputo, la red de soporte en línea, o la recopilación y el procesamiento de datos, todo lo cual representa un costo (Navarro et al., 2018). En Latinoamérica, estos costos no son siempre fáciles de solventar, en gran medida debido a las escasas oportunidades de financiamiento para esta tecnología y a la informalidad imperante en el uso de ésta (Maceira et al., 2010). En cambio, en países de altos ingresos, como Estados Unidos o Canadá, no solo existen mayores oportunidades de financiamiento, sino también un mercado formal y activo para sustentar el desarrollo de los TAI, las PC y las PAM (Jette et al., 2008).

Nuestro objetivo fue determinar la disponibilidad actual de los TAI, las PC y las PAM en Latinoamérica, Estados Unidos y Canadá, para aproximar y comparar el grado actual de desarrollo y la difusión de esta tecnología al servicio de la evaluación psicológica. Nuestra hipótesis es que aún existe una brecha amplia entre lo que está disponible en estas regiones (con desventaja para Latinoamérica cuando se la compara con Estados Unidos y Canadá), pero el tamaño actual de esa brecha es desconocido. Con este estudio pretendemos develar esa brecha. 


\section{Método}

Se realizó un estudio de revisión narrativa (Lozano, 2005) sobre la disponibilidad de los TAI, las PC y las PAM, mediante dos formas de búsqueda independientes: 1) TAI, PC y PAM que aparecen en publicaciones científicas indexadas en bases de datos y 2) TAI, PC y PAM ofrecidos o reportados por editoriales, grupos interesados en el tema y Play Store (Android).

La primera búsqueda se centró en artículos científicos publicados en revistas indexadas en PsycINFO, Scopus, Web of Science, SciELO y PubMed. El propósito fue identificar los TAI, las PC y las PAM que pudieron ser utilizados en tales artículos científicos, ya sea como instrumentos para recolectar datos o como objetos de estudio (v.g. validaciones o estandarizaciones de TAI, PC y PAM). Para lograr dicho propósito, utilizamos descriptores (términos de búsqueda) en inglés y español que permitieran detectar TAI, PC y PAM vinculados a investigaciones realizadas en países latinoamericanos (Argentina, Bolivia, Brasil, Chile, Colombia, Costa Rica, Cuba, Ecuador, El Salvador, Guatemala, Haití, Honduras, México, Nicaragua, Panamá, Paraguay, Perú, República Dominicana, Uruguay y Venezuela) o en Estados Unidos y Canadá (ver anexos I, II y III). La búsqueda se realizó sin definir una fecha límite hacia el pasado. Para Scopus, Web of Science y SciELO se ajustó la búsqueda en el título, el resumen y las palabras clave del autor. Para PubMed se ajustó la búsqueda en el título, el resumen y los términos MeSH. Todos los descriptores fueron revisados y actualizados por un especialista en bibliometría.

La segunda, denominada búsqueda dinámica, se realizó mediante la identificación de TAI y PC ofrecidos o reportados por editoriales, grupos interesados en este tipo de instrumentos. El primer paso fue detectar a los distintos institutos $\mathrm{u}$ organizaciones internacionales, editoriales, grupos de estudios, páginas web dedicadas al uso, el diseño, el desarrollo, la publicación o la distribución de TAI o PC. Estos fueron identificados mediante la búsqueda en literatura gris, así como la navegación en las veinte primeras páginas de Google y Mozilla generadas tras utilizar términos de búsqueda en inglés y español (ver anexo IV). Para los TAI y las PC, una vez ubicadas estas organizaciones, se procedió a enviar correos electrónicos y realizar llamadas a sus contactos, para preguntar directamente por los TAI y las PC que ellos conocían como disponibles o que distribuían actualmente. Asimismo, se exploró en todas las páginas web y los catálogos de pruebas referidos por estos contactos, lo que permitió detectar otros TAI y PC (ver tabla suplementaria I). Con respecto a las PAM, se realizó una búsqueda en Play Store (Android) y fueron identificadas a través de su nombre o título y su descripción general. Luego se procedió a descargar cada PAM de acceso gratuito en un teléfono móvil (Android versión 9) para facilitar la recolección de información detallada.

Los procedimientos de búsqueda dinámica y por publicaciones indexadas las realizaron dos de los in- vestigadores de manera independiente. Luego se cruzaron los datos recolectados por ambos para asegurar la consistencia y que no se perdiera información relevante. Una vez ubicados los TAI, PC y PAM producto de este cruce de búsquedas se registraron varias características de estos instrumentos. Específicamente, detectamos y registramos de cada TAI, PC y PAM su región de procedencia (Estados Unidos, Canadá o Latinoamérica), año de publicación (2014-2019, 2008-2013, 52007), área que evalúa (psicología, salud, educativa, idiomas u otros), población objetivo (adulto mayor de 65 años, adultos, niños y adolescentes, todas), tipo de test o prueba (original, adaptada, no reporta) y si el acceso a los usuarios era libre (sin costo). Toda esta información se registró y organizó en forma de tablas.

\section{Resultados}

Se encontraron 100 TAI, 96 PC y 30 PAM en total. De estos, 84 TAI, 72 PC y 1 PAM fueron detectados en publicaciones en revistas indexadas, mientras que 16 TAI, 24 PC y 29 PAM se identificaron únicamente mediante la búsqueda dinámica. Puede verse un resumen de ambos procedimientos de búsqueda en la Figura 1.

Respecto a los TAI, un $(93.0 \% \mathrm{n}=93)$ fueron publicados o distribuidos en Estados Unidos o Canadá, mientras que solo un $(7.0 \% \mathrm{n}=7)$ en Latinoamérica (Tabla 1). De hecho, la mayoría de los TAI de Latinoamérica $(85.7 \% \mathrm{n}=6)$ fueron publicados entre $2014 \mathrm{y}$ 2019 , lo que es señal de una disponibilidad más reciente que la de Estados Unidos o Canadá $(49.5 \%$ n=46 durante el mismo periodo). Los TAI de Estados Unidos y Canadá son específicamente para evaluación psicológica $(50.5 \% \mathrm{n}=47)$ o de salud en general $(31.2 \% \mathrm{n}=29)$, mientras que en Latinoamérica los temas prioritarios son psicológicos $(42.8 \% \mathrm{n}=3)$ y educativos $(28.6 \% \mathrm{n}=2)$. En ambos contextos, estos instrumentos fueron desarrollados principalmente para la evaluación de adultos (>57.0\%). En Estados Unidos y Canadá, la mayoría de los TAI son productos originales $(82.8 \% \mathrm{n}=77)$, mientras que en Latinoamérica prevalecen las adaptaciones (57.1\% n=4). Sin embargo, en Estados Unidos y Canadá los usuarios deben pagar por la mayoría de los TAI disponibles $(72.0 \% \mathrm{n}=67)$, mientras que en Latinoamérica casi todos los TAI disponibles son gratuitos $(85.7 \%$ $\mathrm{n}=6$ ).

Respecto a las PC, su disponibilidad es mayor en Estados Unidos y Canadá (74.0\% n=71) (Tabla 1). En ambas regiones, la mayoría de las PC han sido publicadas entre 2014 y 2019 (>52\%) y evalúan algún constructo de interés psicológico (>69.0\%). Las PC evalúan a adultos en su mayoría (>56.0\%), en ambas regiones. En Estados Unidos y Canadá, las PC son productos originales en su mayoría $(77.5 \% \mathrm{n}=55)$. Finalmente, las PC tienen costo para los usuarios tanto en Estados Unidos y Canadá (73.3\% n=52) como en Latinoamérica $(52.0 \% \mathrm{n}=13)$.

Respecto a las PAM, Estados Unidos y Canadá registraron la mayor disponibilidad (83.0\% $\mathrm{n}=25)$ (Tabla 1). En ambas regiones, la mayoría de las PAM fueron de- 


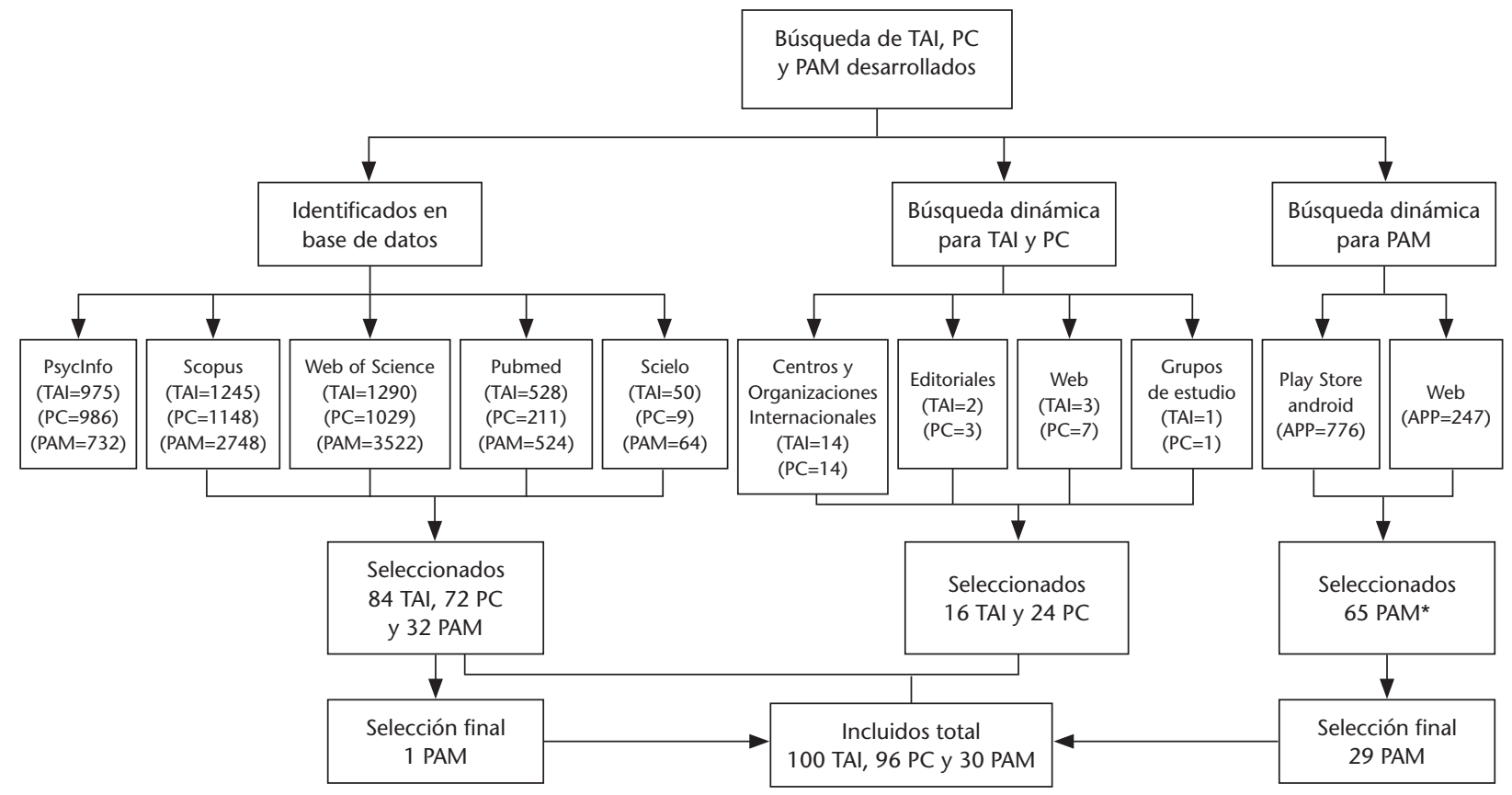

*Se volvió a revisar cada una descargándola en un teléfono android (versión 9) para verificar que su contenido sea una PAM

Figura 1. Diagrama de flujo que resume la metodología de búsqueda del estudio.

Tabla 1. Disponibilidad de los test adaptativos computarizados (TAI), las pruebas computarizadas (PC) y las pruebas en aplicativos móviles (PAM) disponibles en Latinoamérica y en Estados Unidos y Canadá.

\begin{tabular}{|c|c|c|c|c|c|c|}
\hline \multirow[t]{2}{*}{ Disponibilidad según } & \multicolumn{2}{|l|}{ TAI } & \multicolumn{2}{|l|}{ PC } & \multicolumn{2}{|l|}{ PAM } \\
\hline & $\begin{array}{l}\text { Estados Unidos / } \\
\text { Canadá }\end{array}$ & Latinoamérica & $\begin{array}{l}\text { Estados Unidos / } \\
\text { Canadá }\end{array}$ & Latinoamérica & $\begin{array}{l}\text { Estados Unidos / } \\
\text { Canadá }\end{array}$ & Latinoamérica \\
\hline País/Región & $93(93.0 \%)$ & $7(7.0 \%)$ & $71(74.0 \%)$ & $25(26.0 \%)$ & $25(83.0 \%)$ & $5(17.0 \%)$ \\
\hline \multicolumn{7}{|l|}{ Año } \\
\hline 2014-2019 & $46(49.5 \%)$ & $6(85.7 \%)$ & $37(52.1 \%)$ & $13(52.0 \%)$ & $22(88.0 \%)$ & $5(100.00 \%)$ \\
\hline $2008-2013$ & $23(24.7 \%)$ & $1(14.3 \%)$ & $6(8.5 \%)$ & $7(28.0 \%)$ & $3(12.0 \%)$ & $0(00.0 \%)$ \\
\hline$\leq 2007$ & $24(25.8 \%)$ & $0(00.0 \%)$ & $28(39.4 \%)$ & $5(20.0 \%)$ & $0(00.0 \%)$ & $0(00.0 \%)$ \\
\hline \multicolumn{7}{|l|}{ Área que evalúa } \\
\hline Psicología & $47(50.5 \%)$ & $3(42.8 \%)$ & $49(69.0 \%)$ & $20(80.0 \%)$ & $18(72.0 \%)$ & $4(80.00 \%)$ \\
\hline$\underline{\text { Salud }}$ & $29(31.2 \%)$ & $1(14.3 \%)$ & $11(15.5 \%)$ & $3(12.0 \%)$ & $1(4.0 \%)$ & $0(00.0 \%)$ \\
\hline Educativa & $10(10.8 \%)$ & $2(28.6 \%)$ & $9(12.7 \%)$ & $2(8.0 \%)$ & $5(20.0 \%)$ & $1(20.0 \%)$ \\
\hline Idiomas & $7(7.5 \%)$ & $0(00.0 \%)$ & $2(2.8 \%)$ & $0(00.0 \%)$ & $1(4.0 \%)$ & $0(00.0 \%)$ \\
\hline Otros & $0(00.0 \%)$ & $1(14.3 \%)$ & $0(00.0 \%)$ & $0(00.0 \%)$ & $0(00.0 \%)$ & $0(00.0 \%)$ \\
\hline \multicolumn{7}{|l|}{ Población dirigida } \\
\hline Adulto mayor $>65$ & $4(4.3 \%)$ & $0(00.0 \%)$ & $15(21.2 \%)$ & $0(00.0 \%)$ & $0(00.0 \%)$ & $0(00.0 \%)$ \\
\hline Adultos & $70(75.3 \%)$ & $4(57.1 \%)$ & $40(56.3 \%)$ & $15(60.0 \%)$ & $6(24.0 \%)$ & $1(20.0 \%)$ \\
\hline$\underline{\text { Niños y adolescentes }}$ & $15(16.1 \%)$ & $3(42.9 \%)$ & $12(16.9 \%)$ & $6(24.0 \%)$ & $2(8.0 \%)$ & $0(00.0 \%)$ \\
\hline Todas & $4(4.3 \%)$ & $0(00.0 \%)$ & $4(5.6 \%)$ & $4(16.0 \%)$ & $17(68.0 \%)$ & $4(80.00 \%)$ \\
\hline \multicolumn{7}{|l|}{ Tipo de prueba } \\
\hline Original & $77(82.8 \%)$ & $3(42.9 \%)$ & $55(77.5 \%)$ & $5(20.0 \%)$ & $18(72.0 \%)$ & $1(20.0 \%)$ \\
\hline Adaptado & $16(17.2 \%)$ & $4(57.1 \%)$ & $16(22.5 \%)$ & $9(36.0 \%)$ & $7(28.0 \%)$ & $4(80.00 \%)$ \\
\hline No reporta* & $0(00.0 \%)$ & $0(00.0 \%)$ & $0(00.0 \%)$ & $11(44.0 \%)$ & $0(00.0 \%)$ & $0(00.0 \%)$ \\
\hline \multicolumn{7}{|l|}{ Acceso libre } \\
\hline$\underline{\text { Sí }}$ & $26(28.0 \%)$ & $6(85.7 \%)$ & $19(26.7 \%)$ & $12(48.0 \%)$ & $20(80.0 \%)$ & $5(100.00 \%)$ \\
\hline No & $67(72.0 \%)$ & $1(14.3 \%)$ & $52(73.3 \%)$ & $13(52.0 \%)$ & $5(20.0 \%)$ & $0(00.0 \%)$ \\
\hline
\end{tabular}

sarrolladas entre 2014 y 2019 (>88.0\%) y éstas evalúan preferentemente algún constructo de interés psicológico (>72\%). En ambas regiones, la mayoría de PAM $(>68 \%)$ fueron diseñadas para evaluar a poblaciones de todas las edades. En Estados Unidos y Canadá, prevalecen las PAM originales $(72.0 \% \mathrm{n}=18)$. Tanto en Latinoamérica como en Estados Unidos y Canadá, casi todas las PAM son de acceso gratuito para los usuarios $(>80 \%)$.

\section{Discusión}

Realizamos una revisión de la disponibilidad de los TAI, las PC y las PAM en Estados Unidos, Canadá y Latino- 
américa. Los resultados indican que los evaluadores en Estados Unidos y Canadá tienen a su disposición más de trece veces la cantidad de TAI, más del doble de cantidad de PC y el quíntuple de la cantidad de PAM que la que disponen los evaluadores en Latinoamérica. La mayoría de TAI y PC cuentan con un diseño dirigido hacia la evaluación psicológica de adultos, mientras que las PAM suelen estar disponibles para todas las edades. A diferencia de Latinoamérica, en Estados Unidos y Canadá, la mayoría de TAI son de producción original y requieren de un pago para poder ser utilizados. En ambas regiones, el acceso a la mayor parte de las PC disponibles requieren pago para su uso, mientras que la mayoría de PAM en Estados Unidos, Canadá y Latinoamérica no requiere de pago alguno.

La brecha en la disponibilidad de TAI, PC y PAM entre Estados Unidos, Canadá y Latinoamérica es notable y tendría al menos dos posibles causas. La primera corresponde a la comercialización (acceso a TAI y PC previo pago), que permite el financiamiento del desarrollo y la actualización de nuevos TAI y PC (TEA Ediciones, 2019b). Como vimos en este estudio, Estados Unidos y Canadá cuentan con mayor producción de TAI y la mayoría de éstos requieren un pago para su uso, con lo que se brinda así un mayor soporte económico a los desarrolladores. En Latinoamérica, los pocos desarrolladores de TAI generalmente no cobran por sus productos, lo que reduce el soporte económico con el que cuentan para desarrollar nuevas versiones $\mathrm{u}$ otros TAI. En cambio, con las PC ocurre algo distinto: en Latinoamérica hay una mayor proporción que exige pago, lo que estimula la producción de PC y apoya nuevos desarrollos. Para sustentar este punto, nótese que la brecha de PC entre Estados Unidos, Canadá y Latinoamérica es mucho menor que la brecha de TAI. Por supuesto, el fondeo para desarrollo de TAI y PC no es la única desventaja actual de Latinoamérica (regresaremos a este punto más adelante). Respecto a las PAM, la mayoría de acceso gratuito en ambas regiones, recaudan su soporte económico a través de la publicidad comercial que muestran al usuario durante la evaluación (Truong, Nkhoma \& Pansuwong, 2019). Algunas de las PAM encontradas requieren de un cobro adicional para emitir un resultado inmediato y direccionan al usuario hacia páginas web para ofrecer una variada gama de terapias en plataformas en línea. De esta manera, las PAM impulsarían y sostendrían su producción (ver tabla suplementaria IV). No obstante, no queda claro si estas PAM cuentan con confiabilidad y validez verificada (nótese que muy pocas de ellas se encontraron a través de publicaciones científicas), por lo que se recomienda solicitar esa evidencia a los proveedores antes de utilizarlas. La segunda causa posible de la brecha radica en el recurso humano altamente especializado que se necesita para el desarrollo de los TAI, y que todavía no es suficiente en Latinoamérica. Por ejemplo, el entrenamiento en psicometría aplicada -en pregrado y especialmente en posgrado- que puede impulsar una mayor producción de TAI desde la academia es aún muy escaso en Latinoamérica (Rodriguez-
Jimenez et al., 2011). La colaboración científica entre investigadores latinoamericanos y de Estados Unidos y Canadá o de países europeos aún es modesta a este respecto, y supone otra limitación para la producción de TAI, PC y PAM (ver tabla suplementaria III). Desde la empresa privada o las instituciones psicológicas que podrían sumar también con recurso humano especializado y producción de TAI per se, la brecha entre Estados Unidos, Canadá y Latinoamérica sigue siendo grande. Las empresas e instituciones norteamericanas no solo son mayores en número, sino que son más grandes y con más recursos disponibles. Algunos ejemplos son la Educational Testing Service (ETS), la American Educational Research Association (AERA), la Asociación Americana de Psicología (APA) con su división de PsycTests e Ingeniería Pixel, las cuales están dedicadas al desarrollo y la administración de PC, TAI y PAM (ver la tabla suplementaria I para otros ejemplos).

Esta combinación de mayores recursos económicos disponibles y talento humano especializado -y bien organizado- harían una fuerte diferencia, no solo en la adaptación de TAI, PC y PAM, sino también y especialmente en el desarrollo de versiones originales. Como hemos visto, la proporción de TAI, PC y PAM originales de Estados Unidos y Canadá es muy superior a la de Latinoamérica. Acompañando a los factores ya mencionados, una escasa cultura sobre la importancia y el beneficio de las patentes acrecientan el atraso en nuestro contexto latino (Fernandez \& Valera, 2014; Nunes et al., 2013; Rodriguez-Jimenez et al., 2011).

Considerando las fechas de publicación, la producción de TAI, PC y PAM ha aumentado en los últimos cinco años en ambas regiones. Una explicación yace en la masificación de los recursos informáticos e Internet, especialmente en Latinoamérica (Comisión Económica para América Latina y el Caribe, 2016). La masificación hace que a una mayor cantidad de potenciales evaluados -hoy usuarios frecuentes de portátiles, tabletas y teléfonos móviles- les resulte muy familiar responder a cualquier prueba informatizada, con lo que se reducen las barreras tecnológicas que antes representaban una preocupación (Morales-Ramírez et al., 2012; Sternin et al., 2019). El Internet masivo, más confiable y de alta velocidad, facilita hoy no solo el uso de los TAI, las PC y las PAM, sino su distribución y venta. Por ejemplo, varias de estas pruebas pueden comprarse y descargarse en línea de manera inmediata en la web o desde App Play (Apple), Play Store (Android) u otros operativos móviles; por ejemplo, la batería escolar informatizada (TEA Ediciones, 2019a) o la prueba de atención computarizada (Conselho Federal de Psicologia, 2019), con lo que se reducen las barreras de distribución y disminuyen los costos que estas propinaban. Por otro lado, una corriente internacional de evaluación psicológica organizacional que utiliza PC y TAI (Neubert et al., 2015), que introduce algoritmos para la selección de personal o la evaluación de su satisfacción, usando sus resultados como insumos para la toma de decisiones (Chien et al., 2011; Peña-Suárez 
et al., 2017), viene entrando vigorosamente en Latinoamérica en los últimos años. Entre otras cosas, eso explicaría la mayor tendencia de tener a los adultos (edad laboral) como la población-objetivo preferida en ambas regiones. La salud mental (v.g. depresión, ansiedad o adicciones) se explicaría como otro de los tópicos de evaluación preferido debido a la elevada carga de enfermedad que representa en estos países (Organización Mundial de la Salud, 2019). De hecho, las evaluaciones con TAI, PC y PAM ayudan a mejorar notablemente la rapidez y efectividad de los diagnósticos para la detección y prevención en salud mental (Collares \& Cecilio-Fernandes, 2019; Gibbons \& DeGruy, 2019; Graham et al., 2019; Organización Panamericana de Salud, 2017).

\section{Fortalezas y limitaciones}

Como fortaleza, este es el primer estudio de revisión narrativa sobre la disponibilidad de PC, TAI y PAM para evaluadores de Estados Unidos, Canadá y Latinoamérica, y que hace una comparación entre regiones, con la que se pone en evidencia la brecha existente. Entre las limitaciones, no se tuvo acceso completo a varios TAI y PC porque éstos tenían costos altos; sin embargo, eso solo afectó a la precisión del dato sobre si cada prueba era original o adaptada, lo que se considera una limitación menor. Asimismo, nuestro estudio se complementó con una búsqueda dinámica para ubicar aquellos TAI, PC y PAM que se podrían haber encontrado en bases de datos no incluidas. Además, para la inclusión de las PAM, realizamos una revisión individual de todas las PAM de acceso gratuito, a través de su descarga en un teléfono móvil. Esto facilitó una revisión profunda de las de acceso abierto, aunque para las PAM de pago previo solo pudimos alcanzar una revisión básica de su contenido.

\section{Conclusiones}

Los evaluadores en Estados Unidos y Canadá tienen a su disposición más de trece veces la cantidad de TAI, más del doble de la cantidad de PC y el quíntuple de la cantidad de PAM de la que disponen los evaluadores en Latinoamérica. La mayoría de los TAI y las PC está diseñada para evaluar a adultos, aunque las PAM están usualmente disponibles para todas las edades. A diferencia de Latinoamérica, en Estados Unidos y Canadá, la mayoría de TAI es de producción original y requiere de un pago para su uso. El acceso a la mayor parte de las PC disponibles requiere pago, en ambas regiones. A diferencia de Estados Unidos y Canadá, en Latinoamérica, las PAM son usualmente test adaptados y de disponibilidad gratuita.

\section{Conflictos de interés}

Los autores declaran no tener conflicto de intereses

\section{Financiamiento}

El presente estudio fue financiado por la Universidad Católica Los Ángeles de Chimbote.

\section{Agradecimientos}

Queremos agradecer al Mg. Josmel Pacheco-Mendoza, por sus recomendaciones en condición de bibliómetra para este estudio.

\section{Referencias}

Bakker D, Kazantzis N, Rickwood D, Rickard N. (2016). Mental health smartphone apps: review and evidence-based recommendations for future developments. JMIR Ment Health, 3(1), e7. https://doi.org/10.2196/ mental.4984

Burga León, A. (2019). Aplicaciones de la tecnología a la evaluación psicométrica. Propósitos y Representaciones, 7(SPE), e318. http://dx.doi.org/10.20511/pyr 2019.v7nSPE.318

Barney, M., \& Fisher, W. P. (2016). Adaptive measurement and assessment. Annual Review of Organizational Psychology and Organizational Behavior, 3, 469-490. https://doi.org/10.1146/annurev-orgpsych041015-062329

Chien, T.-W., Lai, W.-P., Lu, C.-W., Wang, W.-C., Chen, S.-C., Wang, H.-Y., \& Su, S.-B. (2011). Web-based computer adaptive assessment of individual perceptions of job satisfaction for hospital workplace employees. BMC Medical Research Methodology, 11(1), 47. https://doi.org/10.1186/1471-2288-11-47

Collares, C. F., \& Cecilio-Fernandes, D. (2019). When I say ... computerised adaptive testing. Medical Education, 53(2), 115-116. https://doi.org/10.1111/ medu.13648

Comisión Económica para América Latina y el Caribe. (2016). La nueva revolución digital: De la Internet del consumo a la Internet de la producción. Repositorio digital. https://repositorio.cepal.org/bitstream/handle/11362/38604/4/S1600780_es.pdf

Conselho Federal de Psicologia. (2019). Pruebas completas. SATEPSI. http://satepsi.cfp.org.br/lista_teste_ completa.cfm

Coulacoglou, C., \& Saklofske, D. H. (2018). Psychometrics and psychological assessment. (pp. 3-25). https:// doi.org/10.1016/B978-0-12-802219-1.00001-8

Fernandez, D. A. S., \& Valera, R. M. M. (2014). La actividad innovadora por género en América Latina: Un estudio de patentes. Revista Brasileira de Inovação, 13(1), 163-186. https://doi.org/10.20396/rbi. v13i1.8649075

Ferreri, F., Bourla, A., Mouchabac, S., \& Karila, L. (2018). e-Addictology: An overview of new technologies for assessing and intervening in addictive behaviors. Frontiers in Psychiatry, 9(51), 1-10. https://doi. org/10.3389/fpsyt.2018.00051

Gibbons, R. D., \& DeGruy, F. V. (2019). Without wasting a word: Extreme improvements in efficiency and 
accuracy using computerized adaptive testing for mental health disorders (CAT-MH). Current Psychiatry Reports, 21(8), 67. https://doi.org/10.1007/s11920019-1053-9

Gifford, K. A., Liu, D., Romano, R. R., III, Jones, R. N., \& Jefferson, A. L. (2015). Development of a subjective cognitive decline questionnaire using item response theory: A pilot study. Alzheimer's and Dementia: Diagnosis, Assessment and Disease Monitoring, 1(4), 429-439. https://doi.org/10.1016/j.dadm.2015.09.004 Graham, A. K., Minc, A., Staab, E., Beiser, D. G., Gibbons, R. D., \& Laiteerapong, N. (2019). Validation of the computerized adaptive test for mental health in primary care. The Annals of Family Medicine, 17(1), 23-30. https://doi.org/10.1370/afm.2316

Huang, C.-Y., Tung, L.-C., Chou, Y.-T., Wu, H.-M., Chen, K.-L., \& Hsieh, C.-L. (2018). Development of a computerized adaptive test of children's gross motor skills. Archives of Physical Medicine and Rehabilitation, 99(3), 512-520. https://doi.org/10.1016/j. apmr.2017.07.017

Jette, A. M., Haley, S. M., Ni, P., Olarsch, S., \& Moed, R. (2008). Creating a computer adaptive test version of the late-life function \& disability instrument. The Journals of Gerontology. Series A, Biological Sciences and Medical Sciences, 63(11), 1246-1256. https://doi. org/10.1093/gerona/63.11.1246

Kalender, I., \& Berberoglu, G. (2017). Can computerized adaptive testing work in students' admission to higher education programs in Turkey? Educational Sciences-Theory \& Practice, 17(2), 573-596. https://doi. org/10.12738/estp.2017.2.0280

Lee, S.-C., Huang, Y.-J., Lin, G.-H., Chou, Y.-T., Chou, C.-Y., \& Hsieh, C.-L. (2018). Development of a social functioning assessment using computerized adaptive testing for patients with stroke. Archives of Physical Medicine and Rehabilitation, 99(2), 306-313. https:// doi.org/10.1016/j.apmr.2017.08.492

Lozano, J. M. (2005). De patos, gansos y cisnes. Revisiones narrativas, revisiones sistemáticas y metaanálisis de la literatura. Acta Medica Colombiana, 30(1), 1-3.

Lunz, M. E., Bergstrom, B. A., \& Wright, B. D. (1992). The effect of review on student ability and test efficiency for computerized adaptive tests. Applied Psychological Measurement, 16(1), 33-40. https://doi. org/10.1177/014662169201600103

Maceira, D., Paraje, G., Aramayo, F., Duarte Masi, S., Sánchez, D. (2010). Financiamiento público de la investigación en salud en cinco países de América Latina. Revista Panamericana Salud Publica, 27(6), 442-51.

Morales-Ramírez, A., Escoto, C., García-Lozano, R., Molinar-Solís, J., \& Hidalgo-Cortés, C. (2012). View of sistema para la aplicación de pruebas psicológicas vía web. Acta universitaria, 22(3), 5-13. https://doi. org/10.15174/au.2012.379

Navarro, J.-J., Mourgues-Codern, C., Guzman, E., Rodriguez-Ortiz, I. R., Conejo, R., Sanchez-Gutierrez, C., de la Fuente, J., Martella, D., Saracostti, M. (2018).
Integrating curriculum-based dynamic assessment in computerized adaptive testing: development and predictive validity of the EDPL-BAI battery on reading competence. Frontiers in Psychology, 9, 1492. https:// doi.org/10.3389/fpsyg.2018.01492

Neubert, J. C., Mainert, J., Kretzschmar, A., \& Greiff, S. (2015). The assessment of 21st century skills in industrial and organizational psychology: Complex and collaborative problem solving. Industrial and Organizational Psychology, 8(2), 238-268. https://doi. org/10.1017/iop.2015.14

Nunes, M. A. S. N., Cazella, S. C., Pires, E. A., \& Russo, S. L. (2013). Discussões sobre produção acadêmicocientífica \& produção tecnológica: Mudando paradigmas. Revista GEINTEC - Gestão, Inovação e Tecnologias, 3(2), 205-220. https://doi.org/10.7198/geintec. $\mathrm{v} 3 \mathrm{i} 2.122$

Olea, J., Abad, F. J., \& Barrada, J. R. (2010). Tests informatizados y otros nuevos tipos de tests. Papeles del Psicólogo, 31(1), 94-107.

Olea, J., Ponsoda, V., \& Prieto, G. (1999). Tests informatizados Fundamentos y aplicaciones. Pirámide.

Organización Mundial de Salud. (2019). Salud mental. Organización Mundial de la Salud. https://www.who. int/es/news-room/facts-in-pictures/detail/mentalhealth

Organización Panamericana de Salud. (2017). La salud mental en la Región de las Américas. Mental Health in the Americas. https://www.paho.org/salud-en-lasamericas-2017/?post_t_es=la-salud-mental-en-laregion-de-las-americas

Peña-Suárez, E., Menéndez, F., Eduardo, F.-P., \& Muñiz, J. (2017). Computerized adaptive assessment of organizational climate. Annals of Psychology, 33(1), 152-159. https://doi.org/10.6018/analesps.32.3. 225921

Rodriguez-Jimenez, O. R., Rosero-Burbano, R. F., Botia, M. L., \& Duarte, L. H. (2011). Producción de conocimiento en psicometría en instituciones de educación superior de Bogotá y Chía. Revista Colombiana de Psicología, 20(1), 9-25.

Rojas, A. (2001). Pasado, presente y futuro de los test adaptativos informatizados: Entrevista con Isaac I. Béjar. Psicothema, 13(4), 685-690.

Rose, M., Bjorner, J. B., Fischer, F., Anatchkova, M., Gandek, B., Klapp, B. F., \& Ware, J. E. (2012). Computerized adaptive testing-ready for ambulatory monitoring? Psychosomatic Medicine, 74(4), 338-348. https://doi.org/10.1097/PSY.0b013e3182547392

Shamir, H. (2018). Assessing reading in young learners: using a computerized adaptive reading test for prekindergarten through second grade. Journal of Educational Multimedia and Hypermedia, 27(4), 507-527.

Sierra, F. A., Valdelamar, J. R., Hernández, F. A., \& Sarmiento, L. M. (2009). Test adaptativos informatizados. Avances en Medición, 5, 157-162.

Sternin, A., Burns, A., \& Owen, A. M. (2019). Thirtyfive years of computerized cognitive assessment of aging-Where are we now? Diagnostics, 9(3), 114. https://doi.org/10.3390/diagnostics9030114 
TEA Ediciones. (2019a). BEI. Batería escolar informatizada. TEA Ediciones. http://web.teaediciones.com/ BEI--BATERIA-ESCOLAR-INFORMATIZADA.aspx

TEA Ediciones. (2019b). Historia-Evaluación psicológica, tests psicológicos, psicología, psicometría. Tea Ediciones. http://web.teaediciones.com/Historia.aspx Truong, V. N. X., Nkhoma, M., \& Pansuwong, W. (2019). An integrated effectiveness framework of mobile in-app advertising. Australasian Journal of Information Systems, 23. https://doi.org/10.3127/ ajis.v23i0.1971

Turner-Bowker, D. M., Derosa, M. A., Saris-Baglama, R. N., \& Bjorner, J. B. (2012). Development of a com- puterized adaptive test to assess health-related quality of life in adults with asthma. Journal of Asthma, 49(2), 190-200. https://doi.org/10.3109/02770903.2 011.633674

Van Horn, R. (2003). Computer adaptive tests and computer-based tests. Phi Delta Kappan, 84(8), 567631. https://doi.org/10.1177/003172170308400804 Wang, P., Dai, H., \& Ding, S. (2010). Computerized adaptive testing in Chinese mainland: A review. International Journal of Digital Content Technology and its Applications, 4(6). https://doi.org/10.4156/jdcta. vol4.issue6.11 
Anexo I. Estrategia de búsqueda de artículos que desarrollen TAI

\section{Web of Science}

( $\mathrm{TI}=$ ("computerized adaptive tests" OR "computer test" OR "computerized adaptive testing" OR "computer adaptive version" OR "computer based test")) OR (TS=("computerized adaptive tests" OR "computer adaptive test" OR "computerized adaptive testing" OR "computer adaptive version" OR "computer based test")) OR $(\mathrm{AB}=($ “computerized adaptive tests" OR "computer adaptive test" OR "computerized adaptive testing" OR "computer adaptive version"
$\mathrm{OR}$ "computer based test") $\mathrm{OR}(\mathrm{AK}=($ "computerized adaptive tests" OR "computer adaptive test" OR "computerized adaptive testing" OR "computer

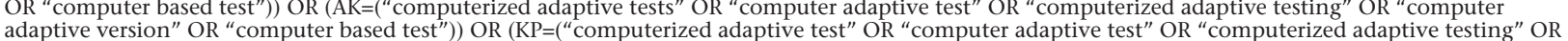
"computer adaptive version" OR "computer based test")) AND ( $(\mathrm{CU}=$ ("Anguilla" OR "Antigua and Barbuda" OR "Argentina" OR "Aruba" OR "Bahamas" "computer adaptive version" OR "computer based test")) AND ((CU=("Anguilla" OR "Antigua and Barbuda" OR "Argentina" OR "Aruba" OR "Bahamas" OR "Colombia" OR "Costa Rica" OR "Cuba" OR "Dominica" OR "Dominican Republic" OR "Republica Dominicana" OR "Ecuador" OR "El Salvador" OR "French Guiana" OR "Grenada" OR "Guadalupe" OR "Guatemala" OR "Guiana" OR "Guyana" OR "Haiti" OR "Honduras" OR "Leeward Islands" OR "Jamaica" OR "Martinique" OR "Mexico" OR "Mejico" OR "Montserrat" OR "Netherlands Antilles" OR "Nicaragua" OR "Panama" OR "Paraguay" OR "Peru" OR "Puerto Rico" OR "Saint Kitts and Nevis" OR "Saint Lucia" OR "Saint Vincent and the Grenadines" OR "Suriname" OR "Surinam" OR "Trinidad and Tobago" OR "Turks and Caicos Islands" OR "United States" OR "United States of America" OR "USA" OR "EE.UU" OR "Uruguay" OR "Venezuela" OR "Virgin Islands of the United States" OR "Windward Islands" OR "Caribbean" OR "Central America" OR "Latin America" OR "South America" OR "West Indies"))

Scopus
TITLE("computerized adaptive test") OR ABS("computerized adaptive test") OR KEY("computerized adaptive test") OR TITLE-ABS-KEY(“Computer Adaptive Test") OR TITLE-ABS-KEY(“Computerized adaptive testing") OR TITLE-ABS-KEY("computer adaptive version") OR TITLE-ABS-KEY("Compute based test") AND AFFILCOUNTRY ("Anguilla" OR "Antigua and Barbuda" OR "Argentina" OR "Aruba" OR "Bahamas" OR "Barbados" OR "Bolivia" OR "Belice" OR "Belize" OR "Brasil" OR "Brazil" OR "British Virgin Islands" OR "Canada" OR "Cayman Islands" OR "Chile" OR "Colombia" OR "Costa Rica" OR "Cuba" OR "Dominica" OR "Dominican Republic" OR "Ecuador" OR "El Salvador" OR "French Guiana" OR "Grenada" OR "Guadalupe" OR "Guatemala" OR "Guiana" OR "Guyana" OR "Haiti" OR "Honduras" OR "Leeward Islands" OR "Jamaica" OR "Martinique" OR "Mexico" OR "Mejico" OR "Montserrat" OR "Netherlands Antilles" OR "Nicaragua" OR "Panama" OR "Paraguay" OR "Peru" OR "Puerto Rico" OR "Saint Kitts and Nevis" OR "Saint Lucia" OR "Saint Vincent and the Grenadines" OR "Suriname" OR "Surinam" OR "Trinidad and Tobago" OR "Turks and Caicos Islands" OR "United States" OR "United States of America" OR "USA" OR "EE.UU" OR "Uruguay" OR "Venezuela" OR "Virgin Islands")

\section{PubMed}

(( (CComputarized adaptative test" OR “Computer Adaptive Test" OR "Computerized adaptive testing" OR "computer adaptive version" OR "Computer based test")) ) AND ("Anguilla" OR "Antigua and Barbuda" OR “Argentina" OR "Aruba" OR "Bahamas" OR "Barbados" OR "Bolivia" OR "Belice" OR "Belize" OR "Brasil" OR "Brazil" OR "British Virgin Islands" OR “Canada" OR "Cayman Islands" OR "Chile" OR "Colombia" OR "Costa Rica" OR "Cuba" OR "Dominica" OR "Dominican Republic" OR "Republica Dominicana" OR "Ecuador" OR "El Salvador" OR "French Guiana" OR "Grenada" OR " "Guadalupe" OR "Guatemala" OR "Guiana" OR "Guyana" OR "Haiti" OR "Honduras" OR "Leeward Islands" OR "Jamaica" OR "Martinique" OR "Mexico" OR "Mejico" OR "Montserrat" OR "Netherlands Antilles" OR "Nicaragua" OR "Panama" OR "Paraguay" OR "Peru" OR "Puerto Rico" OR "Saint Kitts and Nevis" OR "Saint Lucia" OR "Saint Vincent and the Grenadines" OR "Suriname" OR "Surinam" OR "Trinidad and Tobago" OR "Turks and Caicos Islands" OR "United States" OR "United States of America" OR "USA" OR "EE.UU" OR "Uruguay" OR "Venezuela" OR "Virgin Islands of the United States" OR "Windward Islands" OR "Caribbean" OR "Central America" OR "Latin America" OR "South America" OR "West Indies")

\section{PsycINFO}

(Computarized adaptative test /OR Computer Adaptive Test / OR Computerized adaptive testing / OR computer adaptive version / OR Computer based test) AND (argentine/ OR Argentinian/ OR Bolivia/ OR Bolivian/ OR Brazil/ OR Brazilian/ OR Canada/ OR Canadian/ OR Chile/ OR Chilean/ OR Colombia/ OR Colombian/ OR Costa Rica/ OR Costa Rican/ OR Cuba/ OR Cuban/ OR Ecuador/ OR Ecuadorian/ OR The Savior/ OR Salvadoran/ OR Guatemala/ OR Guatemalan/ OR Haiti/ OR Haitian/ OR Honduras/ OR Honduran/ OR Mexico/ OR Mexican/ OR Mejico/ OR Nicaragua/ OR Nicaraguan/ OR Panama/ OR Panamanian/ OR Paraguay/ OR Paraguayan/ OR Peru/ OR Peruvian/ OR Dominican Republic/ OR Dominican/ OR United States/ OR United States of America/ OR USA/ OR EE.UU/ OR Uruguay/ OR Uruguayan/ OR Venezuela/ OR Venezuelan))

(test adaptativo OR prueba adaptativa)

$$
\text { SciELO plataforma general }
$$

\section{Anexo II. Estrategia de búsqueda de artículos que desarrollen pruebas computarizadas}

\section{Web of Science}

(TI=(“computerized tests" OR "computer test" OR "computerized testing" OR "computer version" OR "computer based test")) OR (TS=("computerized tests" OR "computer test" OR "computerized testing" OR "computer version" OR "computer based test")) OR (AB=("computerized tests" OR "computer test" OR "computerized testing" OR "computer version" OR "computer based test")) OR (AK=("computerized tests" OR "computer test" OR "computerized testing" OR "computer version" OR "computer based test")) OR (KP=("computerized tests" OR "computer test" OR "computerized testing" OR "computer version" OR "computer based test")) AND ((CU=("Anguilla" OR "Antigua and Barbuda" OR "Argentina" OR "Aruba" OR "Bahamas" OR "Barbados" OR "Bolivia" OR "Belice" OR "Belize" OR "Brasil" OR "Brazil" OR "British Virgin Islands" OR "Canada" OR "Cayman Islands" OR "Chile" OR "Colombia" OR "Costa Rica" OR “Cuba" OR "Dominica" OR "Dominican Republic" OR "Republica Dominicana" OR "Ecuador" OR "El Salvador" OR "French Guiana" OR "Grenada" OR "Guadalupe" OR "Guatemala" OR "Guiana" OR "Guyana" OR "Haiti" OR "Honduras" OR "Leeward Islands" OR "Jamaica" OR "Martinique" OR "Mexico" OR "Mejico" OR "Montserrat" OR "Netherlands Antilles" OR "Nicaragua" OR "Panama" OR "Paraguay" OR "Peru" OR "Puerto Rico" OR "Saint Kitts and Nevis" OR "Saint Lucia" OR "Saint Vincent and the Grenadines" OR "Suriname" OR "Surinam" OR "Trinidad and Tobago" OR "Turks and Caicos Islands" OR "United States" OR "United States of America" OR "USA" OR "EE.UU" OR "Uruguay" OR "Venezuela" OR "Virgin Islands of the United States" OR "Windward Islands" OR "Caribbean" OR "Central America" OR "Latin America" OR "South America" OR "West Indies"))

Scopus

TITLE(“computerized test”) OR ABS(“computerized test”) OR KEY(“computerized test”) OR TITLE-ABS-KEY("Computer Test”) OR TITLE-ABSKEY(“Computerized testing") OR TITLE-ABS-KEY("computer version") OR TITLE-ABS-KEY("Computer based test") AND AFFILCOUNTRY(“Anguilla" OR "Antigua and Barbuda" OR "Argentina" OR "Aruba" OR "Bahamas" OR "Barbados" OR "Bolivia" OR "Belice" OR "Belize" OR "Brasil" OR "Brazil" OR "British Virgin Islands" OR "Canada" OR "Cayman Islands" OR "Chile" OR "Colombia" OR "Costa Rica" OR "Cuba" OR "Dominica" OR "Dominican Republic" OR "Ecuador" OR "El Salvador" OR "French Guiana" OR "Grenada" OR "Guadalupe" OR "Guatemala" OR "Guiana" OR "Guyana" OR "Haiti" Republic" OR "Ecuador" OR "El Salvador" "OR "French Guiana" OR "Grenada" OR "Guadalupe" OR "Guatemala" OR "Guiana" OR "Guyana" OR " "N "Honduras" OR "Leeward Islands" OR "Jamaica" OR "Martinique" OR "Mexico" OR "Mejico" OR "Montserrat" OR "Netherlands Antilles" OR

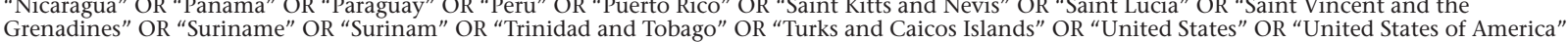
Grenadines" OR "Suriname" OR "Surinam" OR "Trinidad and Tobago" OR "Tur "Tur
OR "USA" OR "EE.UU" OR "Uruguay" OR "Venezuela" OR "Virgin Islands")

\section{PubMed}

((“Computarized test" OR "Computer Test" OR "Computerized testing" OR "computer version" OR "Computer based test"))) AND ("Anguilla" OR "Antigua and Barbuda" OR "Argentina" OR "Aruba" OR "Bahamas" OR "Barbados" OR "Bolivia" OR "Belice" OR "Belize" OR "Brasil" OR "Brazil" OR "British Virgin Islands" OR "Canada" OR "Cayman Islands" OR "Chile" OR "Colombia" OR "Costa Rica" OR "Cuba" OR "Dominica" OR "Dominican "British Virgin Islands" OR "Canada" OR "Cayman Islands" OR "Chile" OR "Colombia" OR "Costa Rica" OR "Cuba" OR "Dominica" OR "Dominican "Guiana" OR "Guyana" OR "Haiti" OR "Honduras" OR "Leeward Islands" OR "Jamaica" OR "Martinique" OR "Mexico" OR "Mejico" OR "Montserrat" OR "Netherlands Antilles" OR "Nicaragua" OR "Panama" OR "Paraguay" OR "Peru" OR "Puerto Rico" OR "Saint Kitts and Nevis" OR "Saint Lucia" OR "Saint Vincent and the Grenadines" OR "Suriname" OR "Surinam" OR "Trinidad and Tobago" OR "Turks and Caicos Islands" OR "United States" OR "United States of America" OR "USA" OR "EE.UU" OR "Uruguay" OR "Venezuela" OR "Virgin Islands of the United States" OR "Windward Islands" OR "Caribbean" OR "Central America" OR "Latin America" OR "South America" OR "West Indies")

\section{PsycINFO}

((Computarized test/ OR Computer Test/ OR Computerized testing/ OR computer version/ OR Computer based test) AND (argentine/ OR Argentinian/ OR Bolivia/ OR Bolivian/ OR Brazil/ OR Brazilian/ OR Canada/ OR Canadian/ OR Chile/ OR Chilean/ OR Colombia/ OR Colombian/ OR Costa Rica/ OR Costa Rican/ OR Cuba/ OR Cuban/ OR Ecuador/ OR Ecuadorian/ OR The Savior/ OR Salvadoran/ OR Guatemala/ OR Guatemalan/ OR Haiti/ OR Haitian/ OI
Honduras/ OR Honduran/ OR Mexico/ OR Mexican/ OR Mejico/ OR Nicaragua/ OR Nicaraguan/ OR Panama/ OR Panamanian/ OR Paraguay/ OR Paraguayan/ OR Peru/ OR Peruvian/ OR Dominican Republic/ OR Dominican/ OR United States/ OR United States of America/ OR USA/ OR EE.UU/ OR Uruguay/ OR Uruguayan/ OR Venezuela/ OR Venezuelan)) 
Anexo III. Estrategia de búsqueda de artículos que desarrollen pruebas en aplicativos móviles

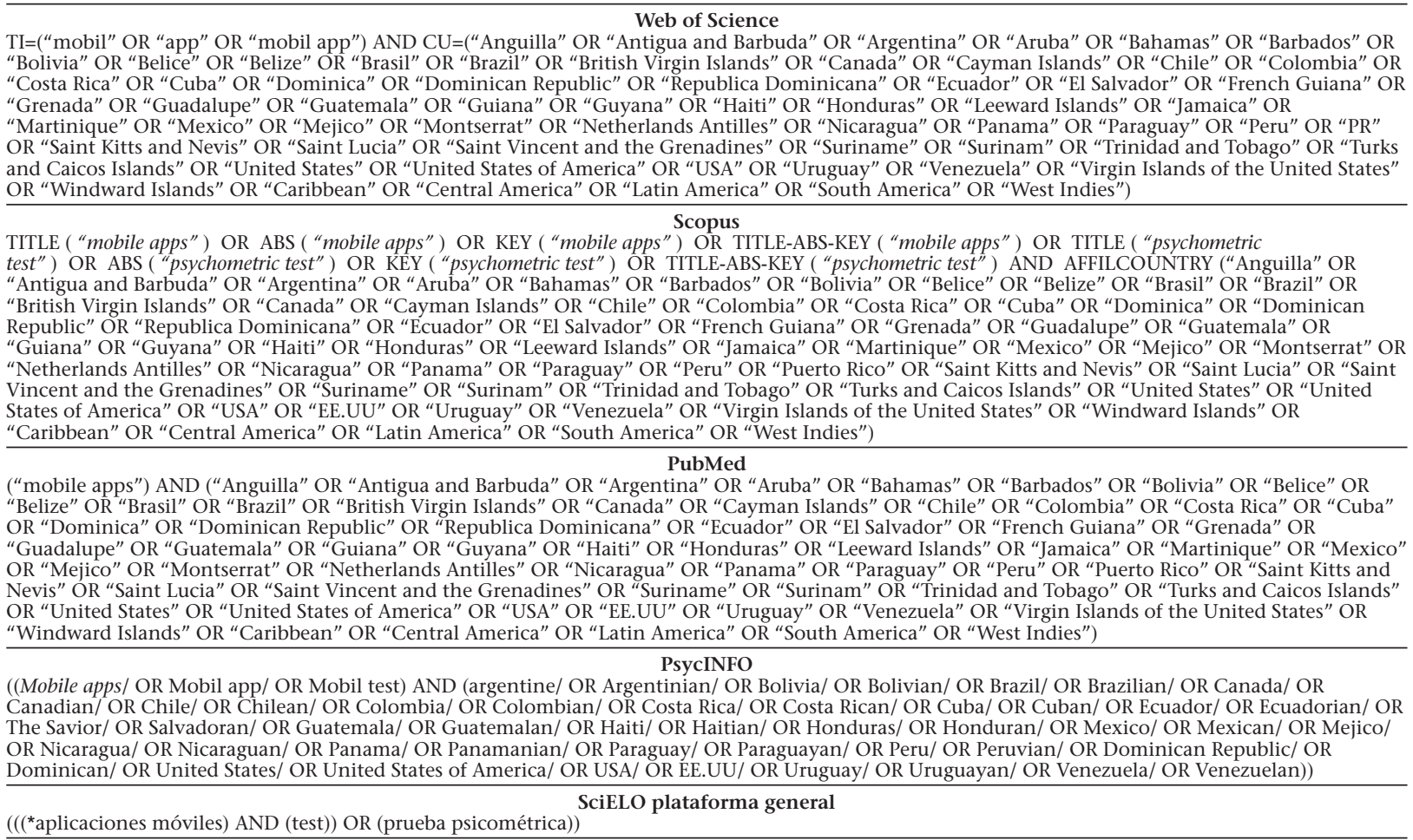

Anexo IV. Términos de búsqueda para TAI/PC/PAM en la web

\begin{tabular}{|c|c|}
\hline \multicolumn{2}{|l|}{ TAI } \\
\hline Inglés & Español \\
\hline $\begin{array}{l}<\text { Computerized adaptive test" OR } \\
\text { "Computer Adaptive Test" OR } \\
\text { "Computerized adaptive testing" } \\
\text { OR "computer adaptive version") }\end{array}$ & $\begin{array}{l}\text { <"Prueba adaptativa computarizada" } \\
\text { OR "Prueba adaptativa de } \\
\text { computadora" OR "versión } \\
\text { adaptativa de computadora") }\end{array}$ \\
\hline \multicolumn{2}{|l|}{ PC } \\
\hline Inglés & Español \\
\hline $\begin{array}{l}<\text { "Computer based test" OR } \\
\text { "Computerized test" OR } \\
\text { "Computer Test" OR } \\
\text { "Computerized testing" OR } \\
\text { "computer version test" OR } \\
\text { "Computer based test"> }\end{array}$ & $\begin{array}{l}\text { <"Prueba basada en computadora" } \\
\text { OR "Prueba computarizada" OR } \\
\text { "Prueba de computadora" OR } \\
\text { "Prueba versión de computadora"> }\end{array}$ \\
\hline \multicolumn{2}{|l|}{ PAM $^{*}$} \\
\hline Inglés & Español \\
\hline $\begin{array}{l}\text { "mobile application tests" OR } \\
\text { "mobile psychological tests" AND } \\
\text { "intelligence test" OR "IQ test" OR } \\
\text { "personality test" OR "vocational } \\
\text { guidance tests" OR "emotional } \\
\text { tests" OR "self-esteem tests" OR } \\
\text { "depression tests" OR "anxiety } \\
\text { tests" OR "psychotechnical tests"> }\end{array}$ & $\begin{array}{l}\text { <"pruebas en aplicativo movil" OR } \\
\text { "pruebas psicologicas moviles" AND } \\
\text { "prueba de inteligencia" OR "prueba } \\
\text { de cociente intelectual" OR "prueba } \\
\text { de personalidad" OR "pruebas } \\
\text { orientación vocacional" OR "pruebas } \\
\text { emocionales" OR "pruebas de } \\
\text { autoestima" OR "pruebas de } \\
\text { depresión" OR "pruebas de } \\
\text { ansiedad" OR "pruebas } \\
\text { psicotécnicas"> }\end{array}$ \\
\hline
\end{tabular}

${ }^{\star}$ Los mismos descriptores fueron usados en Play Store. 
Tabla suplementaria I. Contactados mediante búsqueda dinámica

\begin{tabular}{|c|c|c|}
\hline Nombre & Tipo & Enlace \\
\hline American Psychological Association & Institución & http://bit.ly/39wPHMg \\
\hline APA. Measurement, Evaluation, Statistics, Assessment and Qualitative Inquiry & Institución & http://bit.ly/39k8ACl \\
\hline AERA. Measurement \& Research Methodologies & Institución & http://bit.ly/2wrKCGq \\
\hline American Evaluation Association & Institución & http://bit.ly/2Imca2F \\
\hline Association of Test Publishers & Institución & http://bit.ly/2VNostb \\
\hline Buros Center for Testing & Organización & http://bit.ly/3cta6E4 \\
\hline ERIC Clearinghouse on Assessment and Evaluation (ERIC/AE) & Institución & http://bit.ly/2TFQ13I \\
\hline ERIC (Educational Resources Information Center) & Institución & http://bit.ly/38nvQ0O \\
\hline International Association for Computerized Adaptive Testing & Institución & http://bit.ly/3cpsXQh \\
\hline Psychometric Society & Organización & http://bit.ly/2uVcene \\
\hline ETS Test Collection, Educational Testing Service & Organización & http://bit.ly/39wQdKc \\
\hline PsycTESTS & Organización & http://bit.ly/2IgInbQ \\
\hline Prueba de validez de investigación y evaluación & Grupos de estudio & http://bit.ly/2wnXnlu \\
\hline College Board & Institución & http://bit.ly/2IiVKZc \\
\hline NWEA & Institución & http://bit.ly/2VKI1SH \\
\hline Renaissance Learning & Organización & http://bit.ly/391Dnyy \\
\hline ICFES (Colombia) & Institución & http://bit.ly/2VHnLkM \\
\hline AERA. División de Medición e Investigación & Institución & cmwalker@duq.edu \\
\hline Sistema de Evaluación de Pruebas Psicológicas (SATEPSI) & Editorial & http://bit.ly/2IiG5J7 \\
\hline PEARSON & Editorial & http://bit.ly/2vzS56p \\
\hline TEA EDICIONES & Editorial & http://bit.ly/3amqhBa \\
\hline NCLEX & Web & http://bit.ly/2Tjl1bN \\
\hline CHOIR & Web & https://stanford.io/2wtbLco \\
\hline Ceneval & Web & http://bit.ly/2PKqAhf \\
\hline Evaluar pro & Web & http://bit.ly/2wq3hCC \\
\hline Evaluar & Web & http://bit.ly/2PK3Tty \\
\hline Assess & Web & http://bit.ly/3cmCeZz \\
\hline Education Assessment and Evaluation & Web & http://bit.ly/2Id9btB \\
\hline Ingieneria Pixel & Web & http://bit.ly/2TjTXJp \\
\hline Apkmirror & Web & http://bit.ly/32Lhy90 \\
\hline
\end{tabular}

Tabla suplementaria II. TAI, PC y PAM encontrados

\begin{tabular}{|c|c|c|}
\hline Nombre de la prueba & Acceso libre & Enlace \\
\hline Penn Reading Assessment (PRA-CAT) & no & http://bit.ly/39mbXsr \\
\hline BSS CAT para el riesgo de suicidio & no & http://bit.ly/2TknQcI \\
\hline (HRS-MA) Hansen Research Services Matrix Adaptive Test & no & http://bit.ly/2PDZOar \\
\hline CAT-NUMi & no & http://bit.ly/2ThXjge \\
\hline BODY-Q & no & http://bit.ly/38iRmUC \\
\hline $\begin{array}{l}\text { Prueba adaptativa de computación del inventario de evaluación pediátrica de incapacidad } \\
\text { (PEDI-CAT) }\end{array}$ & no & http://bit.ly/2TkzBQA \\
\hline TBI-CareQOL & no & http://bit.ly/2ImdUcd \\
\hline $\begin{array}{l}\text { BREAST-Q CAT calidad de vida relacionada con la salud y la satisfacción del paciente en la cirugía } \\
\text { de mama }\end{array}$ & no & http://bit.ly/2vAcati \\
\hline (PREM CAT) experiencia informada por el paciente & si & http://bit.ly/38jhazK \\
\hline PEDI-CAT: Dutch versión & no & http://bit.ly/2PGp9jN \\
\hline (CAT-MH) Prueba adaptativa computarizada para la salud mental & si & http://bit.ly/38kzcS4 \\
\hline $\begin{array}{l}\text { EORTC CAT prueba adaptativa por computadora (CAT) de la Organización Europea para la } \\
\text { Investigación y el Tratamiento del Cáncer }\end{array}$ & si & http://bit.ly/38kXdbU \\
\hline calidad de vida en trastornos neurológicos (Neuro-QoL) & no & http://bit.ly/39lyw0m \\
\hline (CAT-MH) adaptativas computarizadas para la salud mental & no & http://bit.ly/2Ifdjt9 \\
\hline EDPL-BAI adaptación dinámica computarizada de procesos de lectura & no & http://bit.ly/3cmDqfv \\
\hline SPQ-CAT Cuestionario de personalidad esquizotípica & no & http://bit.ly/39myfdr \\
\hline WD-FAB Batería de evaluación funcional de discapacidad laboral & no & http://bit.ly/3913OV5 \\
\hline Kid-CAT & no & http://bit.ly/2IiHTBT \\
\hline PEDI-CAT - versión brasileña & si & http://bit.ly/2ThxJYB \\
\hline HDQLIFE Chorea CAT & no & http://bit.ly/2PFMbaE \\
\hline CAT-DI Inventario de depresión de CAT & si & http://bit.ly/2VHpNkU \\
\hline LLFDI-CAT versión holandesa & si & http://bit.ly/2vr5L3K \\
\hline PEDI-SCI AM Medida de actividad de lesión de la médula espinal pediátrica & si & https://go.nature.com/2TASTzL \\
\hline CDI-CAT Inventario de desarrollo comunicativo MacArthur-Bates: Words \& Sentences & no & http://bit.ly/3coZshA \\
\hline AM-PAC-CAT escala ampliada de cognición aplicada & si & http://bit.ly/2wn8Z8m \\
\hline PROMIS versión holandés-flamenco & si & http://bit.ly/38kr1pd \\
\hline PAWB Afecto positivo y el bienestar & si & http://bit.ly/32On1Mc \\
\hline
\end{tabular}


Tabla suplementaria II. TAI, PC y PAM encontrados (Continuación)

\begin{tabular}{|c|c|c|}
\hline Nombre de la prueba & Acceso libre & Enlace \\
\hline SCI-QOL síntomas de ansiedad & si & http://bit.ly/2PLi95n \\
\hline calidad de vida económica de las personas con discapacidad & no & http://bit.ly/2PK5Sy0 \\
\hline (CAT) para el equilibrio & no & http://bit.ly/2TkoTJG \\
\hline Ansiedad y depresión de PROMIS - versión portuguesa & si & http://bit.ly/32SFQOv \\
\hline RAND-IAQL & no & http://bit.ly/2PFMulO \\
\hline CAT-ANX Inventario de ansiedad adaptativo computarizado & si & http://bit.ly/32LFGbH \\
\hline (SSA-BH) Función de salud del comportamiento de la administración del Seguro Social & no & http://bit.ly/2IfdRiH \\
\hline$\underline{\text { RF-CAT función de roles }}$ & no & http://bit.ly/38qjiso \\
\hline (CRIS-CAT) reintegración comunitaria de miembros del servicio lesionados & si & http://bit.ly/3aAwmu7 \\
\hline CAT (EEGS) al examen de ingreso para estudios de posgrado & si & http://bit.ly/2TATgdD \\
\hline (CAT) del TLI índice de responsabilidad transmisible & no & http://bit.ly/2TkAZ5K \\
\hline DMQ-CAT & no & http://bit.ly/2TCpbKH \\
\hline pedsPCF CAT & si & http://bit.ly/32MrOZJ \\
\hline CAT para la disnea & no & http://bit.ly/39lzUjA \\
\hline SWB - CAT calidad de vida & no & http://bit.ly/3aqpAa8 \\
\hline OA-DISABILITY-CAT & no & http://bit.ly/2VHqGtK \\
\hline DA-CAT-2 Actividad diaria de cuidado agudo & no & http://bit.ly/32MVOcR \\
\hline Stress-CAT percepción del estrés & no & http://bit.ly/2uPZbDn \\
\hline OA-FUNCTION-CAT osteoartritis de cadera y rodilla & si & http://bit.ly/32SGuLV \\
\hline CAT FDI the Late-Life & si & http://bit.ly/2uOEoQA \\
\hline Ansiedad-CAT & no & http://bit.ly/2PIiupy \\
\hline Diabetes-CAT & no & http://bit.ly/2TjLXIB \\
\hline CAT-OA osteoartritis & no & http://bit.ly/39gA8IC \\
\hline D-CAT depresión & no & http://bit.ly/2PFNxlK \\
\hline CRF fatiga relacionada con el cáncer & no & http://bit.ly/2Tm69tj \\
\hline CAT-HIT para el dolor de cabeza & no & http://bit.ly/3cvmByK \\
\hline CAT prueba de medicina interna & no & http://bit.ly/2PHtUtw \\
\hline LCAT-25 Ítem & no & http://bit.ly/39kcqeJ \\
\hline CAT-OMI trastornos mentales & no & http://bit.ly/2TjoOG3 \\
\hline LIBRE Profile-CAT impacto de la vida Burn Evaluación de recuperación & no & http://bit.ly/2PLpYIa \\
\hline SPQ-CAT & no & http://bit.ly/39myfdr \\
\hline ThyCAT & no & http://bit.ly/3aswMCH \\
\hline $\begin{array}{l}\text { K-4 students' adaptive } \\
\text { computer-based test for mathematical knowledge and skills }\end{array}$ & si & http://bit.ly/2TlyHTy \\
\hline Prueba de lectura adaptativa por computadora & no & http://bit.ly/2VKLVuP \\
\hline CAT-SS suicido & no & http://bit.ly/2VFNwSB \\
\hline ESI-CAT personalidad & no & http://bit.ly/2PJvOtU \\
\hline ASI-MV Índice de gravedad de adicción - versión multimedia & $\mathrm{si}$ & http://bit.ly/3aAxL3R \\
\hline Modelo de cinco factores (FFM) CAT personalidad & si & http://bit.ly/2VInm1M \\
\hline P-CAT trastorno por estrés postraumático & si & http://bit.ly/32Xk5NL \\
\hline CAT versión of MASS fobia social, TOC, pánico & si & http://bit.ly/2VG9u84 \\
\hline SCI-QO dolor y pérdida & si & http://bit.ly/3aswYlp \\
\hline SCI-FI/AT índice funcional de lesiones & si & http://bit.ly/2IgN1qi \\
\hline MAT-CAT - Herramienta de evaluación de movilidad & no & http://bit.ly/3csauCC \\
\hline CAT para estimar el grado de usabilidad de los sitios de comercio electrónico & si & http://bit.ly/2wq6MsK \\
\hline CAT-DI depresión & si & http://bit.ly/391F1jK \\
\hline A-CAT ansiedad & no & http://bit.ly/391Bh1I \\
\hline FLEX-SF & no & http://bit.ly/2Tyd5m6 \\
\hline Pruebas de matemáticas adaptativas computarizadas & no & http://bit.ly/2ThAsBj \\
\hline Prueba de Memoria Tonal Seashore & no & http://bit.ly/2vCXFEZ \\
\hline (SCD) Prueba adaptativa computarizada para memoria & si & http://bit.ly/3crQ7Wj \\
\hline DAT adaptativo (Pruebas de aptitud diferencial -edición adaptativa computarizada) & no & http://bit.ly/3anofkk \\
\hline WebCAPE (Computer Adaptive Placement Exam) & no & http://bit.ly/32TMPXC \\
\hline $\begin{array}{l}\text { GMAT (Graduate Management Admission } \\
\text { Council) }\end{array}$ & si & http://bit.ly/3ao6ZLJ \\
\hline SNAP-CAT (Programa de personalidad no adaptativa y adaptativa) & no & http://bit.ly/2VJ6lnR \\
\hline NCLEX Examen de licencia para enfermeras & no & http://bit.ly/2TAIjcg \\
\hline CHOIR CAT (Registro de información de resultados de salud colaborativa) & no & https://stanford.io/2wtbLco \\
\hline Prueba GRE CAT & no & http://bit.ly/32KNVog \\
\hline PLAB Pimsleur Idioma Aptitud Batería & no & http://bit.ly/2vr8gDa \\
\hline $\begin{array}{l}\text { COMPASS/ESL (Computerized Adaptive Placement Assessment and Support System and English } \\
\text { as a Second Language) }\end{array}$ & no & http://bit.ly/2TvIl54 \\
\hline ACT ESL Placement Test & no & http://bit.ly/2TCqRDZ \\
\hline$\underline{\text { SBAC (Smarter Balanced assessment consortium) }}$ & no & http://bit.ly/2PHPDBN \\
\hline TOEFL® & no & http://bit.ly/2linf56 \\
\hline MAPS Computerized Placement Test & no & http://bit.ly/2TCqRDZ \\
\hline
\end{tabular}


Tabla suplementaria II. TAI, PC y PAM encontrados (Continuación)

\begin{tabular}{|c|c|c|}
\hline Nombre de la prueba & Acceso libre & Enlace \\
\hline LOEP Levels of English Proficiency & no & http://bit.ly/2TCqRDZ \\
\hline STAR Early Literacy & no & http://bit.ly/38pXHOB \\
\hline SAQ Student Attitude Questionnaire & no & http://bit.ly/2TCqRDZ \\
\hline Facetas de neuroticismo & si & http://bit.ly/2x8o0v9 \\
\hline CAT-WPLT & $\mathrm{si}$ & https://bit.ly/3cAkcU5 \\
\hline CAT-5D-QOL & no & https://bit.ly/30cvjOu \\
\hline PROMIS-D-CAT versión australiana & no & https://bit.ly/309cfjG \\
\hline CP-CAT & no & https://bit.ly/2S68Q0y \\
\hline PEDI - CAT & si & https://bit.ly/36bNxD9 \\
\hline MIRT CAT & no & https://bit.ly/3j8IO8J \\
\hline Brown Location Test & $\mathrm{si}$ & http://bit.ly/2VGOb6s \\
\hline Gibson de habilidades cognitivas- 2 & si & http://bit.ly/38iV8NM \\
\hline (VRFCAT) Virtual Reality Functional Capacity Assessment Tool & si & http://bit.ly/2x7tB4U \\
\hline Batería computarizada Cogstate & no & http://bit.ly/39jPWKB \\
\hline ImPACT & si & http://bit.ly/2VJ78Fl \\
\hline (C-TOC) Cognitive Testing on Computer & no & http://bit.ly/39plFu0 \\
\hline HCT-CV & no & http://bit.ly/2VGHml7 \\
\hline ANAM (métricas de evaluación neuropsicológica automatizada) & no & http://bit.ly/38dQ1hK \\
\hline $\begin{array}{l}\text { CANS-MCI (la pantalla neuropsicológica administrada por computadora para el deterioro } \\
\text { cognitivo leve) }\end{array}$ & si & http://bit.ly/3alQzDJ \\
\hline CNS Vital Signs & si & http://bit.ly/3csFhzu \\
\hline CNTB (batería de prueba neuropsicológica computarizada) & no & http://bit.ly/2VF5HYI \\
\hline CSI (Índice de estabilidad cognitiva) & no & http://bit.ly/3cvyTHt \\
\hline MicroCog & no & http://bit.ly/2wmJfsT \\
\hline Flujos mentales (Neurotrax) & no & http://bit.ly/32Lj3Eg \\
\hline Computerized Neurocognitive & no & http://bit.ly/32OUC9a \\
\hline Computer-based outcome measure of neurology clinical skills & no & http://bit.ly/3aqp8se \\
\hline E computerized Category Test & no & http://bit.ly/39gZx4W \\
\hline ACS computer-based screening test & no & http://bit.ly/38hSqYz \\
\hline VM-REACT (Verbal MemoryRecAll Computerized Test) & no & http://bit.ly/391e3IN \\
\hline PwMS Computerized cognitive batteries multiple sclerosis & no & http://bit.ly/2PIbjOb \\
\hline La batería neurocognitiva computarizada de Penn (PennCNB) & no & http://bit.ly/3aqtS1b \\
\hline (BACS) Breve evaluación de la cognición en la esquizofrenia & no & http://bit.ly/2TCJnMu \\
\hline (RMET) Reading the Mind in the Eyes Test & no & http://bit.ly/32M8ctL \\
\hline $\begin{array}{l}\text { ABCDeti - Prueba de } \\
\text { evaluación de competencia lectora }\end{array}$ & si & http://bit.ly/391Z41w \\
\hline Prueba de inteligibilidad & si & http://bit.ly/2TiFmxW \\
\hline (R-SAT) Herramienta de evaluación de la estrategia de lectura & no & http://bit.ly/2x8JWpZ \\
\hline (CTSC) Prueba computarizada de autocontrol & no & http://bit.ly/32OOGww \\
\hline (BALS en línea) Batería de prueba & no & http://bit.ly/2uRnsZV \\
\hline Prueba de computadora & no & http://bit.ly/2ThZhNv \\
\hline Prueba computarizada TwoDocs & si & http://bit.ly/2TlaahN \\
\hline (ASTB) Batería de prueba de selección de aviación de la Marina de EE. UU. & no & http://bit.ly/2VGapp9 \\
\hline Prueba computarizada (TAVIS) & si & http://bit.ly/2VGxpnS \\
\hline Escala-1- revisada del trastorno de estrés postraumático administrado por un clínico Computarizado & no & http://bit.ly/2TB57IU \\
\hline HERCULES & si & http://bit.ly/2Ij5fY6 \\
\hline (Y-BOCS) Yale-Brown Obsessive-Compulsive Scale & no & http://bit.ly/3avxb7r \\
\hline $\begin{array}{l}\text { computerized information-processing-based } \\
\text { test battery }\end{array}$ & si & http://bit.ly/2TkcWUj \\
\hline Computerized Test of Information Processing (CTIP) & no & http://bit.ly/2ThUThA \\
\hline (GMLT) Cogstate Brief Battery and Cogstate's Groton Maze Learning Test (GMLT) & no & http://bit.ly/2Ihbg7M \\
\hline (LCVA) Low-contrast Visual Acuity & no & http://bit.ly/3cwWRIY \\
\hline (EEG) Electroencephalography & no & http://bit.ly/2vAQOMq \\
\hline (LASSO) Learning About STEM Student Outcomes & si & http://bit.ly/32LqqM5 \\
\hline (GRE) Graduate Record Examination & si & http://bit.ly/39kDuKR \\
\hline (EPT) English Placement Test & no & http://bit.ly/3csTtIF \\
\hline (eSAGE) Self-Administered Gerocognitive Examination & si & http://bit.ly/2VH49gF \\
\hline Nearpod & no & http://bit.ly/3aAWDZh \\
\hline SI-IAT & no & http://bit.ly/3aoBfGh \\
\hline (HHIA) Hearing Handicap Inventory for Adults & no & http://bit.ly/32XKdIl \\
\hline (VRFCAT) Virtual Reality Functional Capacity Assessment Tool & si & http://bit.ly/39oPpqM \\
\hline (APVSAT) Adaptive Paced Visual Serial Attention Task & no & http://bit.ly/2IehTHN \\
\hline MITSI-L & no & http://bit.ly/2IfEe8b \\
\hline (RIA) Ruthven Impairment Assessment & no & http://bit.ly/2VIhJRe \\
\hline NCLEX-RN & no & http://bit.ly/2uUxXf0 \\
\hline (rt-fMRI) Real-time functional magnetic resonance imaging & si & http://bit.ly/38oNIIx \\
\hline
\end{tabular}


Tabla suplementaria II. TAI, PC y PAM encontrados (Continuación)

\begin{tabular}{|c|c|c|}
\hline Nombre de la prueba & Acceso libre & Enlace \\
\hline (WCST-CV-64) Card Sorting Computer Version Test & no & http://bit.ly/2Ifkd1c \\
\hline (DASH) Disabilities of the Arm, Shoulder, and Hand & no & http://bit.ly/2TxyZpq \\
\hline (ANT) Attention Network Task & no & http://bit.ly/2Typ7M5 \\
\hline CNS Vital Signs computerized battery & no & http://bit.ly/2PJ152s \\
\hline Neuropsychological Deficit Scale & no & http://bit.ly/2PGcr4I \\
\hline (AM-PAC) Activity Measure of Post-Acute Care & no & http://bit.ly/2vrvLvR \\
\hline (VRAM) Virtual Radial Arm Maze & no & http://bit.ly/2vpAFtf \\
\hline Hidden Goal Task (HGT) & no & http://bit.ly/38gRBQ1 \\
\hline ROLE-PLAYING GAME (RPG) COMPUTER-BASED TEST & si & http://bit.ly/3aqKJB6 \\
\hline C-TOC & no & http://bit.ly/2vzfW64 \\
\hline $\begin{array}{l}\text { MSPT medición del deterioro y la discapacidad neurológica y neuropsicológica en la esclerosis } \\
\text { múltiple }\end{array}$ & no & http://bit.ly/38qKzby \\
\hline Computer-based test of neurology clinical skills & si & http://bit.ly/3al7wxS \\
\hline Habilidades de neurología de cabecera & si & http://bit.ly/391Qlw7 \\
\hline Test of Analogical Reasoning in Children (TARC) & no & http://bit.ly/3amRTGk \\
\hline Prueba revisada de habilidades cognitivas de Gibson & si & http://bit.ly/38iV8NM \\
\hline L2 para lectura & no & http://bit.ly/38qKHry \\
\hline Prueba de habilidades cognitivas de Gibson & si & http://bit.ly/38iV8NM \\
\hline MSVT Prueba de validez de síntomas médicos & no & http://bit.ly/3cvOaYK \\
\hline basada en computadora de las habilidades neurológicas de cabecera & si & http://bit.ly/391Qlw7 \\
\hline CTIP Prueba computarizada de procesamiento de información & no & http://bit.ly/32KhVAT \\
\hline Prueba de atención computarizada - versión visual (Visual TCA) & no & http://bit.ly/2TNo8YP \\
\hline$\overline{\mathrm{AOL}}$ & no & http://bit.ly/2TNo8YP \\
\hline Escala de calificación de impulsividad - Formas A y B (EsAvI-A y EsAvI-B) & no & http://bit.ly/2TNo8YP \\
\hline HumanGuide & no & http://bit.ly/2TNo8YP \\
\hline $\begin{array}{l}\text { (IDCP-2) Inventario clínico de personalidad Dimensional } 2 \text { y versión de evaluación del inventario } \\
\text { clínico de personalidad dimensional }\end{array}$ & no & http://bit.ly/2TCL8t4 \\
\hline (HPI) inventario de personalidad de Hogan & no & http://bit.ly/3crauCY \\
\hline Raven Advanced Progressive Arrays & no & http://bit.ly/2TCL8t4 \\
\hline (MBTI) Tipos Myers Briggs Type Indicator -Inventario psicológicos & no & http://bit.ly/3crauCY \\
\hline (SMHSC - Del-Prette) Sistema de habilidades sociales multimedia para niños & no & http://bit.ly/2TCL8t4 \\
\hline Sosie $2 .^{\text {a }}$ generación & no & http://bit.ly/2TCL8t4 \\
\hline (TAPMIL) Prueba de aptitud de piloto militar & no & http://bit.ly/2PLDY4V \\
\hline Prueba de inteligencia general no verbal BETA-III (subpruebas y códigos de razonamiento matricial) & no & http://bit.ly/2TCL8t4 \\
\hline (SVV computarizado) Prueba visual subjetiva vertical & si & http://bit.ly/2PLnQjJ \\
\hline CubMemPC: prueba computarizada para evaluar la memoria a corto plazo visoespacial & si & http://bit.ly/2VIO3n0 \\
\hline MemPavox: prueba computarizada para evaluar la memoria de trabajo visoverbal & si & http://bit.ly/2TC8yPo \\
\hline Escala informatizada de evaluación de las características creativas & si & https://bit.ly/3kQeIaG \\
\hline CAMCI & no & https://bit.ly/2EDZhTF \\
\hline Head-Toes-Knees-Shoulders (HTKS) & no & https://bit.ly/36b3Z6x \\
\hline CPMSA & no & https://bit.ly/339fbP2 \\
\hline ASAS & si & https://bit.ly/2EBuiHE \\
\hline WCST Wisconsin Card Sorting Test & si & https://bit.ly/30de1QU \\
\hline Memonum & si & https://bit.ly/30dgU4m \\
\hline Kidtalk's & no & https://bit.ly/2G4aOwa \\
\hline 16 Type Personality Test & si & http://bit.ly/2VDoCmN \\
\hline ADHD Test & si & http://bit.ly/32KyoVJ \\
\hline Bipolar Test & si & http://bit.ly/2IfVOZJ \\
\hline IQ Test & si & https://bit.ly/3dvlcZT \\
\hline IQ Test - Find your IQ! & no & https://bit.ly/3asHRUA \\
\hline IQ Test gratis & si & https://bit.ly/2UFixE9 \\
\hline iQT: Raven IQ Test & si & https://bit.ly/3ds4pqm \\
\hline Know Yourself Personality Test & si & https://bit.ly/2WKbn4d \\
\hline Orientación vocacional & si & https://bit.ly/3amiXpK \\
\hline PsicoTests & si & https://bit.ly/2JpvqNf \\
\hline PTSD Test & si & https://bit.ly/2UnZgIl \\
\hline Test de CI & si & https://bit.ly/2JiifxJ \\
\hline Test de depresión & si & https://bit.ly/330ejy6 \\
\hline Test de personalidad & si & https://bit.ly/2WYM3aX \\
\hline Test de personalidad Big 5 Pro & no & https://bit.ly/2WOK5tA \\
\hline Test Your Anxiety Level & si & https://bit.ly/2xsEEWA \\
\hline Тест: Скоро в школу ! & no & https://bit.ly/2Jgizgq \\
\hline App vocacional & si & http://bit.ly/2PH5XCv \\
\hline Test psicotécnico & si & http://bit.ly/2wRiywH \\
\hline La prueba de color de Lüscher & si & http://bit.ly/2Qbvu7q \\
\hline
\end{tabular}


Tabla suplementaria II. TAI, PC y PAM encontrados (Continuación)

\begin{tabular}{|c|c|c|}
\hline Nombre de la prueba & Acceso libre & Enlace \\
\hline Multiple Intelligences Test & si & http://bit.ly/2Qcfx0y \\
\hline Teste de personalidade científico & si & http://bit.ly/3cWqH3p \\
\hline CI Pro Android & si & https://bit.ly/2UEEQdb \\
\hline Uplift & no & https://bit.ly/3aqq2p0 \\
\hline EncephalApp - Stroop Test & $\mathrm{si}$ & https://bit.ly/39mxlNh \\
\hline اختبار الذكاء IQ Test & $\mathrm{si}$ & https://bit.ly/3igJfNn \\
\hline Alors, c'est quoi? - Learn French in Montreal & si & https://bit.ly/3j8kZhn \\
\hline MindShift CBT - Anxiety Canada & si & https://bit.ly/3czqvaB \\
\hline Histoire du Québec* & no & https://bit.ly/3kSOMNj \\
\hline R2MR & si & https://bit.ly/30eHQAt \\
\hline
\end{tabular}

Tabla suplementaria III. Colaboración internacional entre Latinoamérica, Estados Unidos y Europa

\begin{tabular}{|c|c|c|c|}
\hline Nombre & Colaboración & País Latino & País de colaboración \\
\hline EDPL-BAI adaptación dinámica computarizada de procesos de lectura & si & Chile & Estados Unidos \\
\hline PEDI-CAT - versión brasileña & si & Brasil & Estados Unidos \\
\hline Ansiedad y depresión de PROMIS - versión portuguesa & no & Brasil & - \\
\hline $\begin{array}{l}\text { K-4 students' adaptive } \\
\text { computer-based test for mathematical knowledge and skills }\end{array}$ & no & Chile & - \\
\hline Modelo de cinco factores (FFM) CAT personalidad & si & $\begin{array}{l}\text { República } \\
\text { Dominicana }\end{array}$ & España \\
\hline CAT del grado de usabilidad de los sitios de comercio electrónico & no & Brasil & - \\
\hline Facetas de neuroticismo & no & Argentina & - \\
\hline (RMET) Reading the Mind in the Eyes Test & no & Brasil & - \\
\hline $\begin{array}{l}\text { ABCDeti prueba de } \\
\text { evaluación de competencia lectora }\end{array}$ & no & Colombia & - \\
\hline (BALS en línea) batería de prueba & no & Brasil & - \\
\hline Prueba computarizada TwoDocs & no & Brasil & - \\
\hline Prueba computarizada (TAVIS) & no & Brasil & - \\
\hline ROLE-PLAYING GAME (RPG) COMPUTER-BASED TEST & no & Colombia & - \\
\hline Prueba de atención computarizada - versión visual (Visual TCA) & no & Brasil & - \\
\hline $\mathrm{AOL}$ & no & Brasil & - \\
\hline Escala de calificación de impulsividad - Formas A y B (EsAvI-A y EsAvI-B) & no & Brasil & - \\
\hline HumanGuide & no & Brasil & - \\
\hline $\begin{array}{l}\text { (IDCP-2) Inventario clínico de personalidad dimensional } 2 \text { y Versión de evaluación } \\
\text { del inventario clínico de personalidad dimensional }\end{array}$ & no & Brasil & - \\
\hline (HPI) Inventario de Pprsonalidad de Hogan & no & Brasil & - \\
\hline Raven Advanced Progressive Arrays & no & Brasil & - \\
\hline (MBTI) Tipos Myers Briggs Type Indicator - Inventarios psicológicos & no & Brasil & - \\
\hline (SMHSC - Del-Prette) Sistema de habilidades sociales multimedia para niños & no & Brasil & - \\
\hline Sosie 2. ${ }^{a}$ generación & no & Brasil & - \\
\hline (TAPMIL) Prueba de aptitud de piloto militar & no & Brasil & - \\
\hline $\begin{array}{l}\text { Prueba de inteligencia general no verbal BETA-III (subpruebas y códigos } \\
\text { de razonamiento matricial) }\end{array}$ & no & Brasil & - \\
\hline (SVV computarizado) prueba visual subjetiva vertical & no & Chile & - \\
\hline CubMemPC: prueba computarizada para evaluar la memoria a corto plazo visoespacial & no & México & - \\
\hline MemPavox: prueba computarizada para evaluar la memoria de trabajo visual-verbal & no & México & - \\
\hline App vocacional & no & Perú & - \\
\hline La prueba de color de Lüscher & no & Brasil & - \\
\hline Multiple Intelligences Test & no & Brasil & - \\
\hline Test psicotécnico & no & Perú & - \\
\hline Teste de Personalidade Científico & no & Brasil & - \\
\hline
\end{tabular}


Tabla suplementaria IV. Características de las PAM

\begin{tabular}{|c|c|c|c|c|}
\hline Nombre & $\begin{array}{l}\text { Contiene } \\
\text { anuncios }\end{array}$ & $\begin{array}{l}\text { Resultado } \\
\text { gratuito }\end{array}$ & Fuente de respaldo & $\begin{array}{l}\text { Ofrece otros } \\
\text { servicios }\end{array}$ \\
\hline 16 Type Personality Test & no & si & Indicador Myers-Briggs & no \\
\hline ADHD Test & no & si & Escala de autoinforme TDAH en adultos & si \\
\hline Bipolar Test & no & si & Cuestionario del trastorno del estado de ánimo (MDQ) & si \\
\hline IQ Test & si & si & Teoría de inteligencia Fluida & no \\
\hline IQ Test - Find your IQ* & - & no & - & - \\
\hline IQ Test gratis & si & no & Matrices progresivas de Raven & no \\
\hline iQT: Raven IQ Test & no & si & Matrices progresivas de Raven & no \\
\hline Know Yourself Personality Test & si & si & Especialista en salud (psicólogo) & si \\
\hline Orientación vocacional & si & si & Test de Intereses de Karl Hereford & si \\
\hline PsicoTests & si & si & Especialistas en salud (psicólogos) & si \\
\hline PTSD Test & no & si & Cuestionario PTSD Checklist para el DSM-5 (PCL-5) & si \\
\hline Test de CI & si & si & Matrices progresivas de Raven & no \\
\hline Test de depresión & no & si & Patient Helth Questionnaire (PHQ-9) & no \\
\hline Test de personalidad & si & si & Cuestionario de BIG FIVE & no \\
\hline Test de personalidad Big 5 Pro* $^{*}$ & - & no & Cuestionario de BIG FIVE & - \\
\hline Test Your Anxiety Level ${ }^{* *}$ & si & si & No reporta & si \\
\hline Тест: Скоро в школу* & - & no & - & - \\
\hline App vocacional & no & si & Test de orientación vocacional CHASIDE & no \\
\hline Test psicotécnico** & si & si & No reporta & no \\
\hline La Prueba de Color de Lüscher & si & si & Test de Lüscher & no \\
\hline Multiple Intelligences Test & si & si & Teoría de inteligencias múltiples de Howard Gardner & no \\
\hline Teste de Personalidade Científico & si & si & Teoría de personalidad de Carl Jung & no \\
\hline CI Pro Android & si & si & Matrices progresivas de Raven & si \\
\hline Uplift $^{*}$ & - & - & $\begin{array}{l}\text { PHQ-9 y Generalized Anxiety Disorder Questionnaire } \\
\text { (GAD) }\end{array}$ & - \\
\hline EncephalApp - Stroop Test & no & si & Especialista en salud (psicólogo) & si \\
\hline IQ Test اختبار الذكاء & si & si & No reporta & no \\
\hline $\begin{array}{l}\text { Alors, c'est quoi? - Learn French } \\
\text { in Montreal }\end{array}$ & no & no & No reporta & no \\
\hline MindShift CBT - Anxiety Canada & si & si & Teoría cognitiva conductual & no \\
\hline Histoire du Québec* & - & - & - & - \\
\hline R2MR & no & si & No reporta & no \\
\hline
\end{tabular}

*Las PAM requerían un pago previo y su información fue revisada de su descripción general.

** Los desarrolladores del PAM no reportaron cuál fue su fuente de respaldo, tras consultarse a su correo electrónico. 